Debate / Controversy

\title{
Sondeos electorales 2015 y 2016. Retos metodológicos y estrategias de análisis
}

\section{Electoral surveys 2015 and 2016. Methodological challenges and}

analysis strategies

\section{*Isabel S. Peleteiro Ramos}

ipeleteiro@imop.es

IMOP Insights

Recibido / Received: 14/12/2016

Aceptado / Accepted: 07/03/2017

\section{RESUMEN}

En este texto se reivindica la utilidad social de las encuestas de opinión en la medida en que ayudan a los partidos a diseñar sus propias estrategias de campaña, y proporcionan al potencial votante una fuente de comprensión y de información que le fundamenta su elección. Se insiste, además, en la dificultad de hacer buenos pronósticos debido a los problemas de medida, muestreo y cobertura, abogando por la realización de pronósticos electorales mediante encuestas de calidad con muestras grandes complementadas con investigación cualitativa.

Palabras clave: Encuestas de opinión, industria demoscópica, diseño metodológico, low cost sociológico.

\section{ABSTRACT}

This paper claims the social utility of opinion polls insofar as they help parties to design their own campaign strategies and provide potential voters with a source of understanding and information that underlies their choice. It also stresses the difficulty of making good estimations due to the problems of measurement, sampling and coverage, advocating the realization of electoral forecasts through high quality surveys with large samples complemented with qualitative research.

Key words: Opinion polls, demoscopical industry, methodological design, sociological low cost.

*Autor para correspondencia / Corresponding author: Isabel Peleteiro Ramos. I c./ Antracita, 7 - 4. ${ }^{\circ}, 28045$ Madrid. España/Spain.

Sugerencia de cita / Suggested citation: Peleteiro-Ramos, I. (2017). Sondeos electorales 2015 y 2016. Retos metodológicos y estrategias de análisis. Revista Española de Sociología, 26 (3 Supl.), 123-127.

(http://dx.doi.org/10.22325/fes/res.2016.17) 
Tras los resultados del 26J, las encuestas son, con frecuencia, objeto de guasa y sarcasmo. Aceptando de antemano la evidencia de que efectivamente las encuestas han tenido en España y en otras latitudes en ocasiones desaciertos notables quiero empezar rompiendo una lanza a su favor.

Las encuestas tienen gran validez y relevancia para conocer la opinión de la ciudadanía y su comportamiento declarado. Son útiles para el desarrollo socio-económico del país pero también para el ciudadano de a pie, al que de alguna manera da voz y al que provee de información significativa para formar su opinión. Las empresas de investigación, día a día, hacemos multitud de encuestas para entidades privadas y para la administración pública sobre temáticas diversas: desde la detección de necesidades y demandas sobre la movilidad de los ciudadanos y el consiguiente desarrollo de políticas de transporte público, a los estudios de audiencia que determinan indirectamente el reparto del pastel publicitario, pasando por estudios de satisfacción y calidad de múltiples servicios o sobre hábitos de consumo, por citar solo algunos ejemplos.

Por otro lado, el sector de la investigación de mercados tiene en nuestro país una cifra de negocio neta de 473 millones de euros al año y da empleo a más de 11000 trabajadores, (ANEIMO/ AEDEM0, 2016).

Como cualquier otro sistema de medición las encuestas no están exentas de errores, pero es difícil pensar en la existencia de un método más eficaz para cuantificar y evaluar con precisión lo que opina el conjunto de la ciudadanía, cuáles son sus necesidades y cuáles sus expectativas. En este contexto las encuestas electorales tienen una especial relevancia, ya que no solamente ayudan a los partidos a diseñar sus propias estrategias de campaña sino que es una fuente de comprensión y de información para que el potencial votante tome una decisión más asentada, juiciosa, ponderada y con un mayor conocimiento de causa. Es difícil negar el protagonismo que han tenido las estimaciones electorales en los últimos procesos a tenor de la proyección que le otorgaron los medios de comunicación y los debates ligados a ellas.

Ciertamente, es conocido que las encuestas electorales tienen una dificultad especial: sobre- estiman siempre la participación y tienen un porcentaje de indecisos o de "no sabe, no contesta" claramente mayor que otro tipo de estudios. Por lo tanto, los analistas y los investigadores sociales tenemos que establecer en primer lugar el nivel de participación y luego decidir qué hacer con aquellas personas que no se definen o no nos contestan a las preguntas de intención de voto o de simpatía. No somos adivinos ni jugamos a las quinielas con los resultados, utilizamos procedimientos técnicos que nos aproximan al conocimiento de la realidad, siempre en términos de probabilidad y con un margen de error 0 inexactitud en la estimación.

No todos los factores que explican las diferencias entre los resultados estimados y los resultados reales tienen que ver con la propia encuesta, su diseño y análisis. Y voy más allá, no todas las encuestas pre-electorales pretenden predecir los resultados, al menos no creo que esa sea la prioridad de un responsable de campaña cuando a dos meses de las elecciones solicita un estudio, más bien creo que busca conocer el estado de opinión de los ciudadanos para intentar modificar ese estado a su favor. Efectivamente, la propia campaña, las encuestas y otros elementos externos no siempre previsibles, condicionan 0 afectan al voto. Esto es especialmente importante en un contexto como el actual donde, como hemos visto a lo largo de la campaña, la opinión pública está poco estabilizada y ha mostrado cambios de actitud y de intención de voto de forma relevante entre unas y otras convocatorias. Un somero análisis de los resultados desde diciembre evidencia varios resultados. Una inicial caída de Podemos que remonta una vez que comunicó su unión con Izquierda Unida. Ciudadanos al principio parecía que ascendía, para luego mostrar una tendencia descendente. Y el PP empezó con cierta estabilidad descendente que cambia de sentido hacia el final. Y en esto ha habido una gran coincidencia en todas las encuestas publicadas, es decir, el gran problema en las estimaciones de junio ha sido, básicamente, una infraestimación del voto al Partido Popular y una sobreestimación del voto a Podemos.

Pero hay otra serie de factores vinculados con la encuesta que son en los que me quiero detener. Realizar una buena encuesta no es fácil, ni rápido, 
ni barato. Interpretar adecuadamente los resultados tampoco lo es, y a veces esto parece que se olvida.

Por un lado, los consabidos problemas de medida: sesgos afectados por el diseño del cuestionario, errores del encuestador o del propio encuestado. Aunque a veces es necesario hacer cuestionarios largos para calibrar exactamente cuál es el estado de opinión, a medida que se acercan las elecciones es importante hacer encuestas cortas. Cuanto más corta es la encuesta mayor es la tasa de respuesta, en parte porque la gente es más proclive a participar en el estudio y en parte porque los entrevistadores manifiestan una actitud muchísimo más confiada y proactiva, que se transmite en la forma de presentarse y solicitar la colaboración. Hacer encuestas es cada vez más costoso, cada día se necesitan establecer más contactos para conseguir una entrevista válida. En general en el caso de las encuestas electorales este problema se complica, ya que como los periodos de campo se reducen, la posibilidad de insistir y llamar en varios momentos distintos (incluso los fines de semana) tanto a los "no contesta" como a los contestadores se reduce y por lo tanto tenemos menos posibilidades de contactar con la gente que permanece menos tiempo en sus casas y con los hogares de menor tamaño.

También está la cuestión del tipo de muestreo utilizado. Sabemos que lo ideal es hacer un muestreo probabilístico, pero el muestreo probabilístico a día de hoy es imposible. A través de encuestas personales no es viable porque exige de mucho tiempo y dinero. Tampoco el teléfono es la solución, la permanencia de los individuos en el hogar no es la misma para todos ellos y las negativas tampoco son similares para los distintos segmentos sociales. Podemos diseñar procesos más abiertos, más inclusivos, más complejos..., pero siempre por cuotas, negando así la equi-probabilidad en la selección del individuo.

Luego tenemos claramente problemas de cobertura y aquí podríamos hablar de los marcos muestrales, las encuestas telefónicas limitadas a teléfonos fijos y las encuestas online. Con respecto a los marcos muestrales no me voy a extender mucho, es conocido que no se dispone de ningún marco muestral de teléfonos móviles y que por lo tanto estos tienen que ser generados de manera aleatoria. Es importante describir el procedimiento de obtención de estos teléfonos. También tienen deficiencias muy importantes los marcos muestrales de los teléfonos fijos. Yo me llevé una gran alegría el día que empezaron a aparecer directorios telefónicos digitalizados y que nos libraron de la tortura de grabar los teléfonos que aparecían en las guías telefónicas. Pero inmediatamente el remedio fue peor que la enfermedad y las empresas de telefonía, a diferencia de lo que hacían hasta entonces, empezaron a pedir a sus abonados que decidieran si querían formar parte de ese directorio o no; eso, unido a la incorporación y nuevos usos del teléfono móvil que relega al fijo a un segundo plano, restó valor a poner a disposición del púbico el número de teléfono, de manera que el último directorio que hemos adquirido, proporcionado por la Comisión Nacional de las Telecomunicaciones tiene, aproximadamente, 8000000 de registros frente a los 16000000 de hogares que hay en España. Un buen trabajo exige no solo su codificación y depuración (asignaciones incorrectas de provincia, teléfonos vinculados a una entidad de población o barrio en vez de a un municipio...), sino también complementarlos a través de otros procesos.

No voy a seguir profundizando en ese tema porque me quiero centrar en la falta de cobertura de las encuestas hechas sólo a través de telefonía fija. ¿Nos olvidamos de 1 de cada 4 ciudadanos y seguimos tan tranquilos? Desde la experiencia de haber incorporado los móviles a la investigación en 2004 y haber realizado más de 100000 entrevistas en encuestas pre-electorales, se pone de relieve que los individuos solo móviles tienen un comportamiento — también en lo político — diferente, difícil de corregir solamente a través del equilibraje de la encuesta. Se trata de un dato consistente con otros estudios - y aquí es justo citar a Sara Pasadas y Díaz de Rada que han hecho, en mi opinión, un trabajo importante para resaltar la importancia del teléfono móvil desde lo académico. A lo largo de los últimos 12 años el perfil de los individuos "solo móviles" sufre ciertas modificaciones. Hoy el arquetipo del perfil del "solo móvil" es ligeramente dual. Tenemos por un lado jóvenes profesionales que normalmente viven solos y, por otro 
lado, familias de mayor tamaño, de menor nivel socioeconómico y menor nivel formativo. Además, la penetración y el perfil del "solo móvil" presenta variaciones importantes por CC. AA. que deben ser tenidas en cuenta.

El jueves anterior a las elecciones del 26J hicimos una encuesta de 1300 entrevistas, de las que un $13 \%$ eran "solo móviles". La base es pequeña pero se confirma que el colectivo "solo móvil" tiene menos decidido el voto y muestra menor intención de ir a votar, ha participado menos en otros comicios y manifiesta una mayor propensión de voto al Partido Popular y al Partido Socialista, en detrimento del voto a Podemos y muy especialmente del voto a Ciudadanos, siempre en comparación con los usuarios que tienen fijo y móvil. Por supuesto, el comportamiento de los "solo fijos" también es completamente diferente, pero suponen un volumen de población mucho menor y que en estos momentos no estamos dejando fuera de la encuesta en ningún caso. Destaco estos resultados porque hacen referencia a las últimas elecciones, pero la mayor intención de voto al Partido Popular por parte de los entrevistados a través de móviles para el conjunto del Estado es una constante que se repite en estudios realizados en distintos momentos.

Me gustaría también hablar del tamaño de las muestras, quizás revelen que en esta ocasión todos nos confiamos en que el trabajo era más fácil. El tamaño medio de las encuestas que se publicaron la última semana "publicable" fue de 1450 casos, para el mismo periodo ese tamaño fue de 3429 en las elecciones del 20 de diciembre, más del doble. Tal vez pecamos de atrevidos, ya que tenemos poca experiencia sobre repetición de elecciones en un corto periodo de tiempo, creo que solamente se cuenta con las experiencias del "tamayazo" de Madrid y de Asturias en 2012, y tanto en un caso como en el otro el panorama difería notablemente del actual. El margen de error publicado, en torno a los 3 puntos porcentuales, no deja de ser una convención, puesto que en primer lugar no trabajamos con muestreos probabilísticos y además las estimaciones y las ponderaciones que se llevan a cabo no solamente incrementan este error sino que son difíciles de medir. Pero fundamentalmente el problema reside en que con estos tamaños muestrales es inviable profundizar en las posibles divergencias que se producen dentro de cada uno de los colectivos de potenciales votantes de un partido 0 coalición. En unos momentos de tanta volatilidad y donde la base social de cada partido no está consolidada, estudiar los movimientos internos es importante.

Es muy probable que en esta ocasión unos y otros hayamos sido incapaces de estimar o calibrar adecuadamente el grado de abstención. Quizá no fuimos capaces de prever que se iba a dar en la medida en que se dio en Podemos, ya que incluso entre los jóvenes - que es el segmento tendencialmente menos propenso a ir a votar-, eran los de Podemos con diferencia los que se declaraban más inclinados a acudir a las urnas. Hubo un inadecuado ajuste de los "no sabe" y los "no contesta" y por eso infra-estimamos la intención de voto al Partido Popular.

Una de las hipótesis que se han planteado, a posteriori, era la posibilidad de que los votantes de Podemos más descontentos con los cambios, con la unión con Izquierda Unida, con la estrategia desarrollada por el partido y por su líder en los últimos tiempos, no participasen tan activamente en las encuestas. Hicimos el esfuerzo de escuchar a posteriori todas las negativas y curiosamente encontramos un perfil femenino, muy rural y de gente mayor que a priori se corresponde fundamentalmente con el perfil del votante del PSOE y del Partido Popular. Sin duda, habría que seguir insistiendo en este aspecto.

¿Y ahora qué? Pues con las limitaciones que hoy impone el mercado parece deseable aquilatar los resultados, explicar su significado y alcance, insistir en que las encuestas hacen estimaciones dentro de unos márgenes de probabilidad, primero por cómo se obtienen dichas estimaciones, y segundo porque la realidad puede cambiar. Lo que están diciendo las encuestas en un momento puede reflejar una realidad que varía al cabo de una semana.

El crédito de una encuesta debería basarse en el diseño metodológico, en el proceso de recogida de datos, en el cuestionario utilizado, en el tamaño muestral y por la información que proporciona: qué 
ha hecho, cómo lo ha hecho y qué resultados ha obtenido.

Y en este contexto creo que la sociedad en su conjunto, los académicos y los profesionales del sector, tenemos que trabajar para que las encuestas preelectorales tengan la suficiente calidad. Me parece muy importante que demos, entre todos, valor a la investigación electoral, que presentemos encuestas sólidas, más sólidas que las de ahora, y que en la medida de lo posible demos datos en continuidad, que se puedan hacer investigaciones continuas y que nos apoyemos en la investigación cualitativa, ya que quizás sea la técnica más adecuada para analizar conductas emergentes y para detectar nuevas tendencias. Y, sobre todo, que trabajemos con muestras más grandes que nos permitan analizar con mayor detalle la variabilidad intraelectores de cada grupo político. En cualquier caso las encuestas deben cumplir con las recomendaciones de las asociaciones y de los colegios profesionales y de la propia Ley Electoral de publicar la ficha técnica. Incluso debemos dar un paso más y al igual que en otros países de nuestro entorno que se ofrezcan siempre los datos de intención directa y de recuerdo de voto.

Lo barato a veces sale caro, el low cost no funciona en investigación social y de Santa Bárbara también hay que acordarse cuando no truena. 
